\title{
CMCF-ID: A versatile high-flux and micro-focusing beamline for macromolecular crystallography
}

\author{
Kiran Mundboth, Michel Fodje, Shaun Labiuk, Kathryn Janzen, James Gorin, and Pawel Grochulski \\ Canadian Light Source Inc., University of Saskatchewan \\ kiran.mundboth@lightsource.ca
}

The Canadian Macromolecular Crystallography Facility (CMCF) at the Canadian Light Source Inc. is composed of two beamlines; the CMCF-ID which utilizes the intense $x$-rays from an insertion device and the CMCF-BM which employs the radiation emitted from a bending magnet.

The CMCF-ID beamline has been operating successfully for the past 12 years with worldwide users from a diverse array of scientific backgrounds. Both beamlines are specialized in high-resolution diffraction studies enabling structural characterization of single crystals of proteins and nucleic acids as well as small molecules and microcrystalline samples.

High-flux, high-resolution and fast data collection even on micron-sized $(<5 \mu \mathrm{m})$ samples will soon become the new standard in macromolecular crystallography. To keep up with these new challenges and satisfy the ever-increasing demands of users, the CMCF-ID beamline is undergoing a major upgrade of its components.

Primarily, the current insertion device will be replaced by a more powerful one that will quadruple the photon source flux density while a new monochromator consisting of a pair of $\mathrm{Si}$ (111) crystals and a pair of multilayers will allow more advanced techniques including serial crystallography experiments. Depending on the monochromatization mode, photon fluxes of $1 \mathrm{e} 14 \mathrm{ph} / \mathrm{s}$ (Si crystals) and up to $1 \mathrm{e} 15 \mathrm{ph} / \mathrm{s}$ (multilayers) are expected at the sample position.

Three elliptical mirrors placed at specific locations along the $x$-ray beam path will successively condition the $x$-ray beam both in the horizontal and vertical directions. The mirrors will be bendable (i.e. varying radii of curvature) and therefore the beam can be focused at the sample position modifying its size accordingly to match the dimensions of the crystal being studied. An adjustable vertical beam size ranging from 5 to $50 \mu \mathrm{m}$ is expected while horizontally the beam will be set to $50 \mu \mathrm{m}$.

As the first phase of the upgrade in 2017, two new components have already been integrated into the beamline, namely, the sample changer (ISARA robot) and a Pilatus $6 \mathrm{M}$ area detector. With the new robot, a sample exchange cycle is completed in $<25$ seconds while the detector is facilitating shutter-less operation. These have resulted in efficiently decreasing data collection time by at least a factor of 5 . We plan to further this success and fully upgrade the beamline by the end of 2021. 